\title{
Diffusion Tensor Imaging of the Uterine Zones Related to the Menstrual Cycle and Menopausal Status at 3 Tesla MRI
}

\author{
Özgür Kılıçkesmez ${ }^{1}$, Zeynep Fırat ${ }^{2}$, Ayşegül Oygen², Duygu Kara Bozkurt² ${ }^{2}$, Tevfik Güzelbey², \\ Bengi Gürses ${ }^{2}$, Neslihan Taşdelen ${ }^{2}$
}

${ }^{1}$ Department of Diagnostic and Interventional Radiology, İstanbul Training and Research Hospital, İstanbul, Turkey

${ }^{2}$ Department of Radiology, Yeditepe University Medical School, İstanbul, Turkey

Background: Diffusion and diffusion tensor imaging techniques (DTI) are widely available and used both in central nervous system and body imaging, including gynecological diseases.

Aims: The aims of this study were to assess the capability of DTI of uterine zones in relation to the menstrual cycle and ascertain the normal apparent diffusion coefficient and fractional anisotropy values at 3T magnetic resonance imaging (MRI).

Study Design: Prospective clinical study.

Methods: A total of 13 young reproductive and 12 postmenopausal healthy volunteers were included in the study. MRI examination included sagittal T2-weighted and singleshot echo planar imaging DTI obtained under free breathing. Fractional anisotropy (FA) values of the endometrium, junctional zone, and myometrium were determined.

Results: The median (minimum-maximum) FA of the endometrium, myometrium, and junctional zone of the reproductive group were 0.31 (0.260-0.465), 0.42 (0.302-0.664), and $0.58(0.420-0.745)$, respectively, in the proliferative phase and $0.26(0.180-0.413), 0.48(0.357-0.656)$, and $0.59(0.490-0.675)]$, respectively, in the secretory phase. In the postmenopausal group, the FA values of the endometrium, myometrium, and junctional zone were 0.275 (0.136-0.425), 0.255 (0.191-0.553), and 0.27 (0.129-0.397), respectively. Apparent diffusion coefficient (ADC) values of the endometrium, myometrium, and junctional zone of the reproductive group were $1.25 \pm 0.254$ (0.970-1.463), 1.67 (1.213-1.854), and 1.23 (0.853-1.301), respectively, in the proliferative phase and $1.32 \pm 0.283(1.165-1.706), 1.55$ (1.360-1.791), and $1.17(1.163-1.705)$, respectively, in the secretory phase. In the postmenopausal group, the ADC values of the endometrium, myometrium, and junctional zone were measured as $1.100 \pm 0.192(0.850-1.302), 1.14(0.864$ $1.283)$, and 1.09 (0.912-1.291). The FA values of the endometrium and myometrium were lower in the secretory phase of the reproductive group, while ADC values were higher. However, both the FA and ADC values were lower in the postmenopausal group.

Conclusion: The present study showed that uterine DTI is feasible when used quantitatively. While FA values tend to decrease, ADC values increase significantly in all zones in the secretory phase except the junctional zone. Zonal FA and $\mathrm{ADC}$ values of postmenopausal women are lower in comparison to those in young women.

Keywords: Diffusion tensor imaging, fractional anisotropy, uterus, menstrual cycle
Magnetic resonance imaging (MRI) is a well-known diagnostic tool for the assessment of different gynecological diseases, owing to its superior soft tissue contrast (1). The excellent demonstration of uterine zonal anatomy with T2weighted MR sequences is a well-known entity, and MRI is a widely used radiological technique for detecting uterine pathologies. However, the thickness and signal intensities of the myometrium and especially the endometrium change during different phases of the menstrual cycle $(2,3)$. Depending on the histologic changes in the myometrium and endometrium, 
variation of the apparent diffusion coefficient (ADC) values of the normal uterine zones in the different periods of the cycle is demonstrated in the literature $(4,5)$. Diffusion-weighted imaging (DWI) can show abnormal signals reflecting pathologic foci based on differences in molecular diffusion. It also allows for quantitative evaluation of the ADC that may be useful for the differentiation of malignant and benign tissues, and for demonstrating therapeutic outcomes $(6,7)$.

Diffusion tensor imaging (DTI) may be defined as advanced DWI, which can be used to determine the water diffusion directionality in detail. Diffusion is not a one-dimensional process, and in the organized viscera, diffusion does not occur equally in all directions. The directionality of water diffusion is defined by anisotropy index (fractional anisotropy), which determines microstructural arrangement, like the quantity and direction of fibrous tissue $(8,9)$. In tissues like the kidneys, due to the presence of collecting ducts and tubules, or the uterus, due to the muscle structures, diffusion has anisotropic properties. Diffusion tensor imaging is the best method to evaluate the anisotropic properties of solid organs, and it, unlike DWI, permits the analysis of diffusion along multiple directions. Recently, the improvement of this technique made it a useful and complementary sequence to conventional imaging techniques for demonstrating the fiber structures of the uterus $(4,8,10-13)$.

Knowledge of the fractional anisotropy (FA) values of normal uterine zones will be required during the quantitative evaluation of DTI images in various diseases of the uteri of women at different menstrual cycle phases and ages. The purpose of this study was to define zonal uterine DTI parameters in premenopausal healthy volunteers in relation to the menstrual cycle, along with post-menopausal healthy women, and to evaluate the feasibility of the technique. Although the endometrium is not composed of fiber structures, we aimed to determine the FA values for comparison with the other zonal structures.

\section{MATERIALS AND METHODS}

\section{Study population}

The local ethics committee approved the study, and informed consent was signed by all subjects. From June 2013 to November 2014, a total of 13 healthy volunteers in the premenopausal reproductive period with a median (minimummaximum) age of 28 (25-33), and a total of 12 postmenopausal women with a median (minimum-maximum) age of 50 (age range: 48-65) were enrolled in the study. Among premenopausal women, exclusion criteria included irregular menstrual cycles, oral contraceptive usage, gynecological disease history, cesarean section history, and pathological findings on
MRI examination. The median (minimum-maximum) menstrual cycle duration was 28 days (27-31 days). The volunteers were chosen among women with regular cycles for the last six months. Among postmenopausal women, exclusion criteria were postmenopausal bleeding, gynecological disease history, hormone replacement therapy, and abnormal MRI findings. In the postmenopausal study group, MRI examination was performed once. On the other hand, MRI examinations were performed twice in the reproductive group, including the midproliferative and mid-secretory phases. The menstrual period included the first four days of the cycle, the proliferative period started after the fourth day, and the secretory period included the days after ovulation, which was assumed to occur on the $14^{\text {th }}$ day. All reproductive women underwent pelvic MRI in the mid-proliferative period ( $9^{\text {th }}$ day of the cycle) and the mid-secretory period $\left(21^{\text {st }}\right.$ day the cycle).

\section{Magnetic resonance imaging protocol}

MRI was performed with a 3T MRI system (Intera Achieva, Philips Medical Systems, Philips Healthcare; Best, Netherlands), including high-power gradients with a slew rate of $200 \mathrm{mT} / \mathrm{m} /$ ms and a maximum strength of $80 \mathrm{mT} / \mathrm{m}$. A six-channel phased array SENSE Torso coil was used in examinations. For each patient, a turbo spin echo T2-weighted (TSE) sequence in the sagittal plane and a sagittal DTI were performed using the single-shot (SS) echo planar imaging (EPI) technique with free breathing.

Diffusion tensor imaging using SS EPI (slice thickness, 3 $\mathrm{mm}$; interslice gap, $1 \mathrm{~mm}$; repetition time (TR), $10000 \mathrm{~ms}$; echo time (TE), $60 \mathrm{~ms}$; matrix, $100 \times 132$; voxel size, $1.67 \times 1.67 \times 3.00$ $\mathrm{mm}$; acquisition time, $6.12 \mathrm{~min}$; number of excitations (NEX), 2; number of slices, 40; b, 0 and $700 \mathrm{~s} / \mathrm{mm}^{2}$ ) was acquired in the sagittal plane with free breathing. The parallel imaging method was used with a sensitivity encoding for a fast MRI (SENSE) factor of 2. The diffusion direction number was 16 . The field of view (FOV) was $260 \mathrm{~mm}$, and the thickness and interslice gap values were the same for DTI and T2W images to enable anatomical compatibility for analysis.

\section{Data analysis}

After acquisition of the images, the data were transferred to a workstation equipped with manufacturer-supplied software (Extended MR Workspace R 2.6.3.1, Philips Healthcare; Best, Netherlands) for analysis. Initially, the T2-weighted images were evaluated to exclude the presence of uterine pathology and whether the three zones of the uterus were clearly discernible. These evaluations and subsequent DTI measurements were performed by two experienced radiologists (BG: 8 years, OK: 13 years) in body diffusion imaging. In the postmenopausal group, one subject with poor discrimination of uterine zones was excluded. 
A T2-weighted sequence was used as an anatomical guide while drawing regions of interest (ROI) on the $b=0$ images. Freehand ROIs were placed on the target zone to include whole pixels at the chosen slices on the endometrium, junctional zone, and myometrium. Although the junctional zone is less discernible in the postmenopausal period, we measured it by tracing the innermost part of the myometrium.

For DTI data analysis and measurements, the midsagittal and two parasagittal slices were used to include the most proper area of each uterine layer, and hence the largest possible ROI by selecting the midsagittal three slices. The special software automatically measured the FA and ADC of the chosen ROIs. Measurements were done in each zone by two radiologists in agreement. These measurements were done for each reproductive group subject, in the mid-proliferative and mid-secretory periods. For the postmenopausal group, the MRI examination was performed once, and the FA and ADC measurements were carried out with the same technique as in the premenopausal group. For each measurement, great attention was paid to not putting ROIs on the areas with susceptibility artifacts. Angular threshold and anisotropy values were in the range of 30-40 and 0.21-0.20, respectively. Also, colorcoded FA maps were created in subjects, and the blue color represented craniocaudal, green color antero-posterior, and red color medio-lateral dimensions of diffusion. The strength of the anisotropy was shown by the intensity of the colors.

\section{Statistical analysis}

Statistical analysis was done with a commercially available software (Statistical Package for Social Sciences version 18, SPSS Inc.; Chicago, IL, USA). The differences between the zonal measurements of three different groups (proliferative, secretory, and postmenopausal) were evaluated using a Kruskal-Wallis and post-hoc tests and Bonferroni correction. The adjusted alpha value was 0.017 , and the significance level was 0.003 . Then, within-group differences were assessed with the Wilcoxon signed-rank test. The comparisons between the pre- and post-menopausal groups were performed with the Mann-Whitney U test. P values less than 0.05 were considered statistically significant.

\section{RESULTS}

The age distribution of the two groups was significantly different $(\mathrm{p}<0.001)$. The median (minimum-maximum) FA values of endometrial zone in the proliferative and secretory phases of the reproductive group and postmenopausal group were $0.31(0.260-0.465), 0.26(0.180-0.413)$, and 0.275 (0.136-0.425), respectively. The difference was statistically significant between the proliferative and secretory groups $(p=0.011)$. The median (minimum-maximum) ADC values of the endometrial zone were measured as $1.25 \pm 0.254(0.970$ $1.463), 1.32 \pm 0.283(1.165-1.706)$, and $1.100 \pm 0.192(0.850$ 1.302) $\left(\times 10^{-3} \mathrm{~mm}^{2} / \mathrm{s}\right)$, respectively, and the difference was statistically significant between the proliferative and secretory groups $(\mathrm{p}=0.010)$.

The median (minimum-maximum) FA values of the myometrium in the proliferative and secretory phases and the postmenopausal period were $0.42(0.302-0.664), 0.48(0.357$ $0.656)$, and 0.255 (0.191-0.553), respectively. The ADC values were measured as 1.67 (1.213-1.854), 1.55 (1.360-1.791), and $1.14(0.864-1.283)\left(\times 10^{-3} \mathrm{~mm}^{2} / \mathrm{s}\right)$, respectively. The difference was statistically significant between the reproductive and postmenopausal groups for both FA $(\mathrm{p}<0.001)$ and ADC $(\mathrm{p}<0.001)$ values.

In the proliferative phase, the median FA value of all three zones were higher compared with the secretory phase. Both proliferative and secretory FA values of the endometrium and myometrium were higher when compared with postmenopausal measurements.

$\mathrm{ADC}$ values tend to increase significantly in all zones in the secretory phase except the junctional zone. Like FA, ADC also differed with age. In the postmenopausal period, ADC values decreased significantly for the endometrium and myometrium $(\mathrm{p}<0.001)$.

The median FA and ADC measurements and the statistical findings are summarized in Tables 1 and 2. Graphs of endometrial FA and ADC values are shown in Figure 1-3. Representative sagittal T2 sequence and the corresponding FA maps of the secretory phase of the uterus in a reproductive volunteer and a postmenopausal volunteer, along with a demonstration of ROI placement, are shown in Figure 4, 5.

\section{DISCUSSION}

In this study, we were able to obtain uterine DTI images that were free of artifacts (which was evaluated qualitatively) and measure zonal FA values in all three zones both in the reproductive and postmenopausal periods in vivo with a 3T MRI machine. In the postmenopausal period, however, the junctional zone was less discernible from the neighboring zones.

Fiocchi et al. (10) showed that the FA and ADC values of the whole uterus in a cesarean-scarred uteri group were $0.42 \pm 0.02$ and $1.82 \pm 0.18$, respectively. For comparison, the volunteers' values were similar, without a statistical difference: $0.41 \pm 0.02$ and $1.93 \pm 0.25$. The measurements of anterior isthmic regions revealed a difference in fiber density and numbers between 
TABLE 1. Median (minimum-maximum) zonal and cyclic FA and ADC values of the reproductive (proliferative and secretory) and the postmenopausal groups

\begin{tabular}{|c|c|c|c|c|c|c|}
\hline & \multicolumn{2}{|c|}{ Endometrium } & \multicolumn{2}{|c|}{ Myometrium } & \multicolumn{2}{|c|}{ Junctional Zone } \\
\hline & FA & $\begin{array}{c}\mathrm{ADC} \\
\left(\mathrm{x} 10^{-3} \mathrm{~mm}^{2} / \mathrm{s}\right)\end{array}$ & FA & $\begin{array}{c}\mathrm{ADC} \\
\left(\mathrm{x} 10^{-3} \mathrm{~mm}^{2} / \mathrm{s}\right)\end{array}$ & FA & $\mathrm{ADC}$ \\
\hline Proliferative phase $(\mathrm{n}=13)$ & $\begin{array}{c}0.31 \\
(0.260-0.465)\end{array}$ & $\begin{array}{c}1.25 \pm 0.254 \\
(0.970-1.463)\end{array}$ & $\begin{array}{c}0.42 \\
(0.302-0.664)\end{array}$ & $\begin{array}{c}1.67 \\
(1.213-1.854)\end{array}$ & $\begin{array}{c}0.58 \\
(0.420-0.745)\end{array}$ & $\begin{array}{c}1.23 \\
(0.853-1.301)\end{array}$ \\
\hline Secretory phase $(n=13)$ & $\begin{array}{c}0.26 \\
(0.180-0.413)\end{array}$ & $\begin{array}{c}1.32 \pm 0.283 \\
(1.165-1.706)\end{array}$ & $\begin{array}{c}0.48 \\
(0.357-0.656)\end{array}$ & $\begin{array}{c}1.55 \\
(1.360-1.791)\end{array}$ & $\begin{array}{c}0.59 \\
(0.490-0.675)\end{array}$ & $\begin{array}{c}1.17 \\
(1.163-1.705)\end{array}$ \\
\hline $\mathrm{p}$ (Proliferative versus secretory) & 0.011 & 0.010 & 0.080 & 0.033 & 0.408 & 0.649 \\
\hline Post-menopausal (n=12) & $\begin{array}{c}0.275 \\
(0.136-0.425)\end{array}$ & $\begin{array}{c}1.100 \pm 0.192 \\
(0.850-1.302)\end{array}$ & $\begin{array}{c}0.255 \\
(0.191-0.553)\end{array}$ & $\begin{array}{c}1.14 \\
(0.864-1.283)\end{array}$ & $\begin{array}{c}0.27 \\
(0.129-0.397)\end{array}$ & $\begin{array}{c}1.09 \\
(0.912-1.291)\end{array}$ \\
\hline $\begin{array}{l}\text { p (Median productive versus post-menopau- } \\
\text { sal) }\end{array}$ & 0.574 & $<0.001$ & $<0.001$ & $<0.001$ & $<0.001$ & 0.728 \\
\hline
\end{tabular}

TABLE 2. FA values of the endometrium in the reproductive and postmenopausal patients

\begin{tabular}{|c|c|c|c|c|c|}
\hline & & Proliferative P. & Secretory P. & & Postmenopausal \\
\hline $\mathrm{Nr}$ & Age & FA & FA & Age & FA \\
\hline 1 & 33 & 0.31 & 0.30 & 65 & 0.42 \\
\hline 2 & 29 & 0.33 & 0.22 & 50 & 0.13 \\
\hline 3 & 31 & 0.46 & 0.31 & 52 & 0.27 \\
\hline 4 & 25 & 0.28 & 0.27 & 48 & 0.36 \\
\hline 5 & 28 & 0.41 & 0.41 & 49 & 0.22 \\
\hline 6 & 31 & 0.26 & 0.29 & 48 & 0.44 \\
\hline 7 & 27 & 0.32 & 0.25 & 46 & 0.30 \\
\hline 8 & 25 & 0.28 & 0.22 & 48 & 0.23 \\
\hline 9 & 32 & 0.28 & 0.32 & 49 & 0.17 \\
\hline 10 & 25 & 0.31 & 0.26 & 53 & 0.22 \\
\hline 11 & 27 & 0.32 & 0.22 & 55 & 0.24 \\
\hline 12 & 30 & 0.30 & 0.24 & 48 & 0.30 \\
\hline 13 & 29 & 0.29 & 0.18 & & \\
\hline Mean & 29 & 0.32 & 0.27 & 51 & 0.28 \\
\hline
\end{tabular}

FA: fractional anisotropy; $\mathrm{Nr}$ : subject number; $\mathrm{P}$ : phase

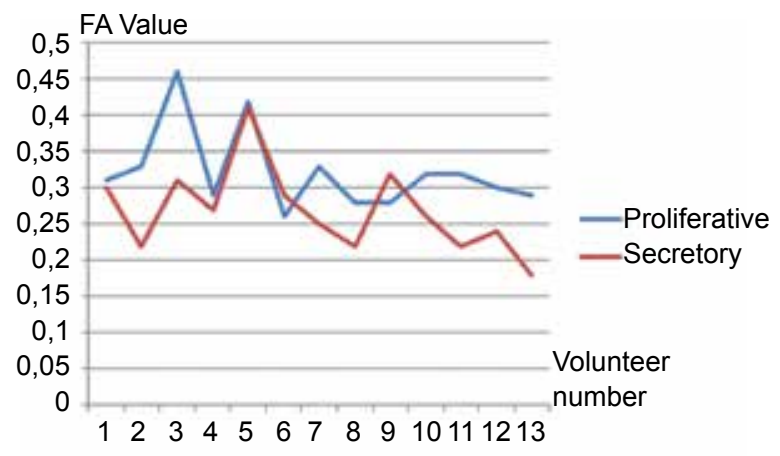

FIG. 1. Graph showing the cyclic endometrial FA values of the reproductive group. (FA: fractional anisotropy)

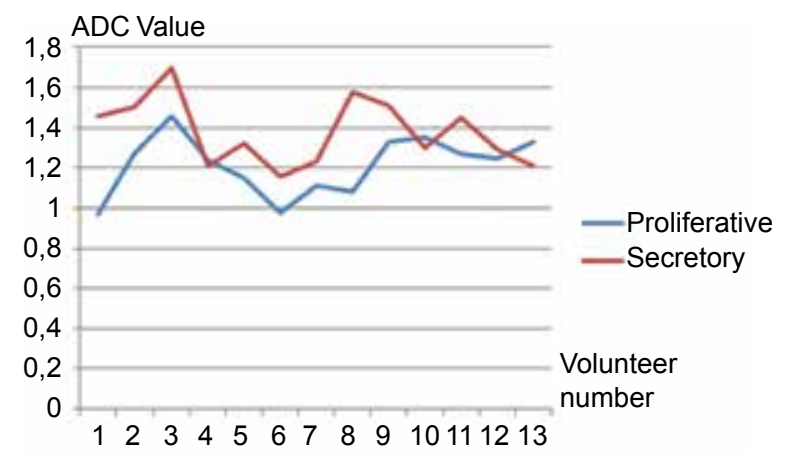

FIG. 2. Graph showing the cyclic endometrial ADC values of the reproductive group. (ADC: apparent diffusion coefficient)

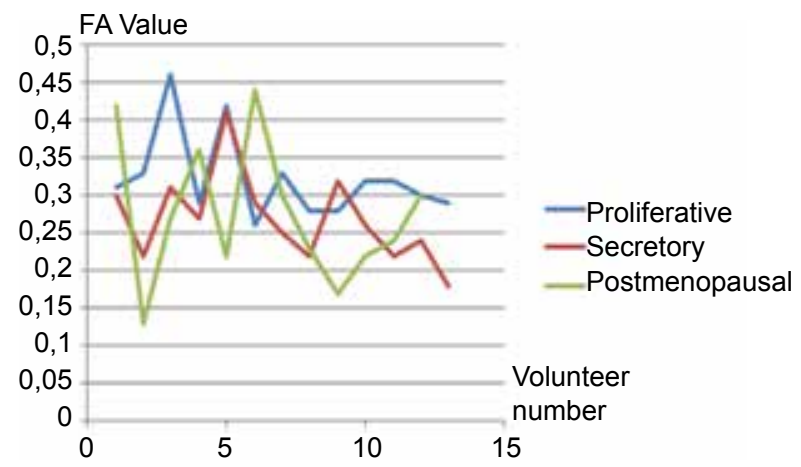

FIG. 3. Graph showing the endometrial FA values of the reproductive and postmenopausal groups. (FA: fractional anisotropy)

these two groups, which were smaller in the cesarean section group. The study was carried out on a 3T MRI, and DTI was obtained with a b value of $\left(0.600 \mathrm{smm}^{-2}\right)(10)$. In an ex vivo study published by Toba et al. (8), data were obtained from five ex vivo organs of patients subjected to hysterectomy for medical reasons. In this study, a 4.7T MRI was used, and the ADC values of the interior and exterior myometrial zones and tumor were not statistically different. The FA values of the interior 

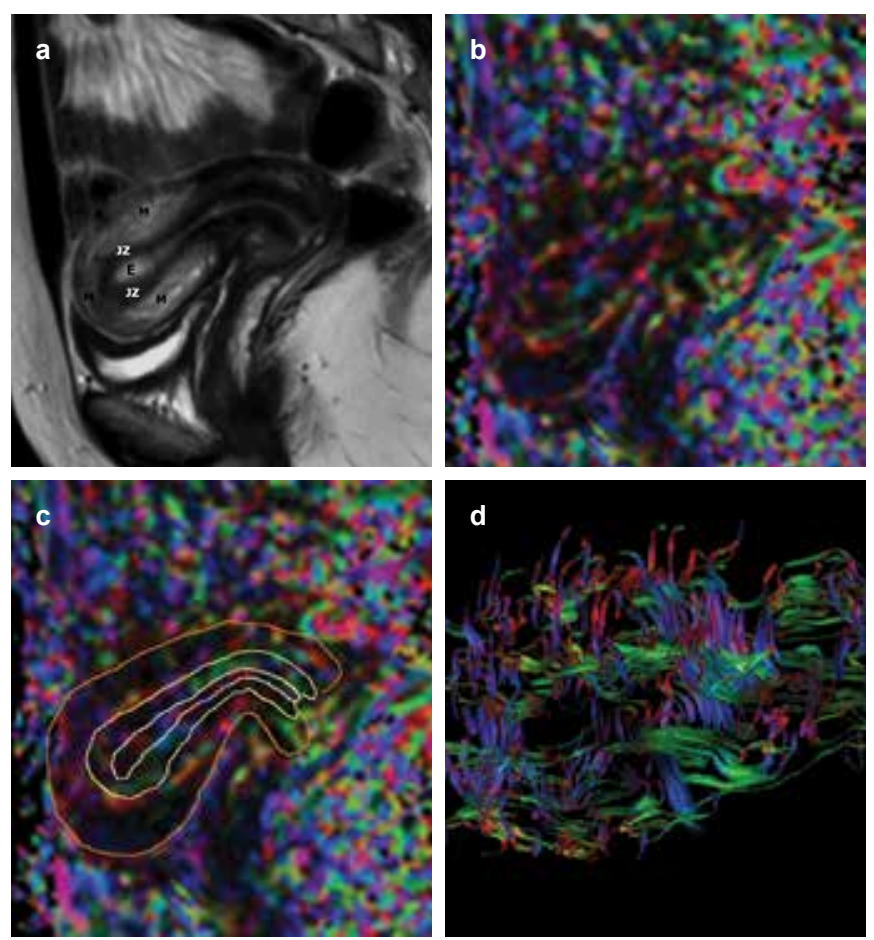

FIG. 4. a-d. Sagittal MRI of the uterus of a 28 -year-old volunteer in the secretory phase; a sagittal T2-weighted image shows the zonal differentiation with great detail and resolution (E: endometrium, the central white line; JZ: junctional zone, dark grey line encircling the endometrium; M: myometrium, the thick outer light grey muscle tissue) (a), and the sagittal color-coded fractional anisotropy map shows low zonal differentiation (b). Region of interest insertion for zonal measurements (area confined into white line: endometrium; area between yellow and white lines: junctional zone; area between orange and yellow lines: myometrium) (c) and sagittal 3D tractography image of the uterus (blue fibers: circular fibers; green fibers: longitudinal fibers) (d).
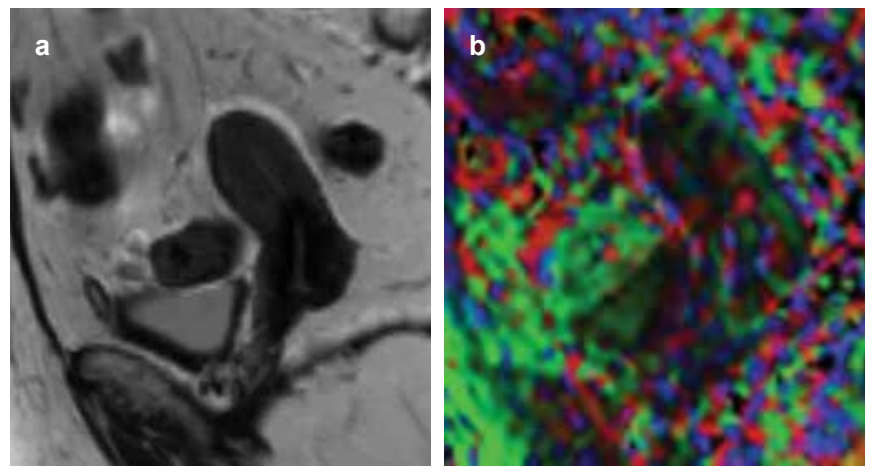

FIG. 5. a, b. Sagittal MRI of the uterus of a 53-year-old volunteer; the sagittal T2-weighted image shows the poor zonal differentiation and thinner endometrium compared with younger volunteers $(a)$, and the sagittal-color coded fractional anisotropy map (b).

$(0.446 \pm 0.01)$ and exterior myometrial zones $(0.326 \pm 0.08)$ were statistically higher than the tumor $(0.216 \pm 0.05)(\mathrm{p}<0.01$ for both) (8). Thrippleton et al. (14) performed an ex vivo study with a 7T MRI and found the FA value of the leiomyo- mas to be around $0.20-0.28 \pm 0.053$, and the myometrium as $0.395 \pm 0.032$; they concluded that these findings suggest that water molecule diffusion may be fastest in a direction parallel with fibers of the myometrial muscles and dense fibroid structures (14).

The differences in the FA and ADC values of the cyclic reproductive and postmenopausal endometrium are likely to determine variations in the viscosity of the interstitial space and in the ratio of the volume occupied by cells to the volume of the interstitial space. The amount of water molecules decreases in the postmenopausal period, so the lower FA and higher $\mathrm{ADC}$ values of the endometrial zone in the younger women may be answered by increased estrogen, progesterone, and gonadotropins. With increasing age, the age-dependent changes in the $\mathrm{ADC}$ of the endometrial zone indicate that the age factor should be taken into account during quantitative evaluation of endometrial abnormalities $(15,16)$. Also, with increasing age, the FA values of the myometrium and junctional zone showed a decreasing tendency, while the FA values of the endometrium were almost unchanged, indicating the differentiation of uterine microstructural organization, such as the density and orientation of fibrous tissue, as age increased. As widely known, endometrial thickness increases in the later phases of the menstrual cycle in correlation with serum levels (17). A similar correlation between estradiol and the changes of endometrial FA values may imply that higher estradiol results in a higher isotropy of water diffusion directionality and lower FA values (18).

Studies regarding the cyclic ADC changes of uterine zones in the literature revealed that ADC values increase beginning from the proliferative phase to secretory phase, and are higher in the secretory phase, probably related to increased fluid content $(4,15,19,20)$.

A study by Kuang et al. (15) revealed that the endometrial ADCs in different age groups were higher in the midsecretory period than those during the mid-proliferative pe$\operatorname{riod}(\mathrm{p}<0.05)$. There was no statistical difference between two periods or three age groups for the myometrial and the junctional zones.

Kido et al. (4) found that the ADC values of the endometrium were $1.00 \times 10^{-3} \mathrm{~mm}^{2} / \mathrm{s}$ in the menstrual phase, which increased to $1.41 \times 10^{-3} \mathrm{~mm}^{2} / \mathrm{s}$ in the mid-proliferative phase and then to $1.45 \times 10^{-3} \mathrm{~mm}^{2} / \mathrm{s}$ in the secretory phases. Similarly, Tsili et al. (20) found a difference in the zonal ADC values in different phases. Endometrial ADC values were calculated as $1.39 \pm 0.20 \times 10^{-3} \mathrm{~mm}^{2} / \mathrm{s}$ for the proliferative phase, $1.50 \pm 0.18 \times 10^{-3} \mathrm{~mm}^{2} / \mathrm{s}$ for the secretory phase, and $1.25 \pm 0.27$ $\mathrm{x} 10^{-3} \mathrm{~mm}^{2} / \mathrm{s}$ for the menstrual phase (20). Our results are also in concordance with the existing reports; endometrial ADC in the mid-proliferative phase increased from $1.21 \pm 0.25$ to $1.38 \pm 0.28 \mathrm{~mm}^{2} / \mathrm{s}$ in the mid-secretory period. 
In the mid-proliferative period, glandular, endometrial stromal, and the endothelial cells of the vessels showed a significant proliferation as a reaction to increased estrogen. These cells included large nuclei with undeveloped cytoplasms, which resulted in the restriction of water molecules' diffusivity both in the extracellular and intracellular spaces. In the mid-secretory period, increased progesterone leads to secretion in the endometrium, which results in stromal focal necrosis, glandular atrophy, and edema secondary to epithelial glandular coiling. These mechanisms may be the cause of the high ADC values of the endometrial zone during the mid-secretory phase (15,21-23). For the myometrium, edema in the secretory phase may have an effect to lower FA values, whereas myometrial contractions and relatively low water content might have increased FA values (4).

Like the other reports, the present study also demonstrated a significant zonal difference of ADC values and with some of the FA values $(4,5,15,19,20)$.

This study includes some limitations. First, we performed DTI in the two periods of the menstrual cycle and excluded the menstrual period. Nevertheless, the blood products in the endometrial cavity may influence the reliability of the measurements in the menstrual phase. Second, the subject number was relatively low.

In conclusion, the present study shows that quantitative DTI of the uterine zones is technically possible with 3T MRI. FA values of the uterus vary depend on zonal structure, age, and the period of the menstrual cycle. While FA values tend to decrease, ADC values significantly increase in all zones in the secretory phase except in the junctional zone. With aging, both FA and ADC values decrease. During interpretation of the FA values of the uterus, the age, zone, and periods of the menstrual cycle should be considered.

Ethics Committee Approval: Ethics committe approval for this study was received from the ethics committee of Yeditepe University School of Medicine.

Informed Consent: Written informed consent was obtained from the patients who participated in this study.

Peer-review: Externally peer-reviewed.

Author contributions: Concept - O.K., Z.F., N.T.; Design - O.K., B.G., N.T.; Supervision - O.K., N.T., B.G.; Resource - D.K.B., A.O.,T.G.; Materials - D.K.B.,T.G., A.O.; Data Collection and/or Processing - D.K.B., T.G., A.O.; Analysis and/or Interpretation - Z.F., D.K.B., T.G.; Literature Search - Z.F., D.K.B.,T.G.; Writing - O.K., B.G., Z.F.; Critical Reviews - O.K., B.G., Z.F.

Conflict of Interest: No conflict of interest was declared by the authors.

Financial Disclosure: The authors declared that this study has received no financial support.

\section{REFERENCES}

1. Koyama T, Togashi K. Functional MR imaging of the female pelvis. J Magn Reson Imaging 2007;25:1101-12. [CrossRef]

2. Haynor DR, Mack LA, Soules MR, Shuman WP, Montana MA, Moss AA. Changing appearance of the normal uterus during the menstrual cycle: MR studies. Radiology 1986;161:459-62. [CrossRef]

3. Hricak H. MRI of the female pelvis: a review. AJR Am J Roentgenol 1986;146:1115-22. [CrossRef]

4. Kido A, Kataoka M, Koyama T, Yamamoto A, Saga T, Togashi $\mathrm{K}$. Changes in apparent diffusion coefficients in the normal uterus during different phases of the menstrual cycle. Br J Radiol 2010;83:524-8. [CrossRef]

5. Kilickesmez O, Bayramoglu S, Inci E, Cimilli T, Kayhan A. Quantitative diffusion-weighted magnetic resonance imaging of normal and diseased uterine zones. Acta Radiol 2009;50:340-7. [CrossRef]

6. Charles-Edwards EM, deSouza NM. Diffusion-weighted magnetic resonance imaging and its application to cancer. Cancer Imaging 2006;6:135-43. [CrossRef]

7. Namimoto T, Awai K, Nakaura T, Yanaga Y, Hirai T, Yamashita Y. Role of diffusion weighted imaging in the diagnosis of gynecological diseases. Eur Radiol 2009;19:745-60. [CrossRef]

8. Toba M, Miyasaka N, Sakurai U, Yamada I, Eishi Y, Kubota T. Diagnostic possibility of diffusion tensor imaging for the evaluation of myometrial invasion in endometrial cancer: an ex vivo study. J Magn Reson Imaging 2011;34:616-22. [CrossRef]

9. Bammer R, Acar B, Moseley ME. In vivo MR tractography using diffusion imaging. Eur J Radiol 2003;45:223-34. [CrossRef]

10. Fiocchi F, Nocetti L, Siopis E, Currà S, Costi T, Ligabue G, et al. In vivo $3 \mathrm{~T}$ MR diffusion tensor imaging for detection of the fibre architecture of the human uterus: a feasibility and quantitative study. Br J Radiol 2012;85:1009-17. [CrossRef]

11. Yonglan H, Ding N, Xue HD, Jin Z, Ha S, Cao J. Cyclic changes of the uterus and cervix in young and middle-aged women during the menstrual cycle: an initial 3T MR functional imaging study based on T2 mapping and diffusion tensor imaging (DTI) sequences. ECR 2014 DOI: 10.1594/ecr2014/C-0498.

12. Weiss S, Jaermann T, Schmid P, Staempfli P, Boesiger P, Niederer $\mathrm{P}$, et al. Three-dimensional fiber architecture of the nonpregnant human uterus determined ex vivo using magnetic resonance diffusion tensor imaging. Anat Rec A Discov Mol Cell Evol Biol 2006;288:84-90. [CrossRef]

13. Fujimoto K, Kido A, Okada T, Uchikoshi M, Togashi K. Diffusion tensor imaging (DTI) of the normal human uterus in vivo at 3 tesla: comparison of DTI parameters in the different uterine layers. J Magn Reson Imaging 2013;38:1494-500. [CrossRef]

14. Thrippleton MJ, Bastin ME, Munro KI, Williams AR, Oniscu A, Jansen MA, et al. Ex vivo water diffusion tensor properties of the fibroid uterus at $7 \mathrm{~T}$ and their relation to tissue morphology. J Magn Reson Imaging 2011;34:1445-51. [CrossRef]

15. Kuang F, Ren J, Huan Y, Chen Z, Zhong Q. Apparent diffusion coefficients of normal uterus in premenopausal women with 
3.0-T magnetic resonance imaging. J Comput Assist Tomogr 2012;36:54-9. [CrossRef]

16. Burger HG, Hale GE, Dennerstein L, Robertson DM. Cycle and hormone changes during perimenopause: the key role of ovarian function. Menopause 2008;15:603-12. [CrossRef]

17. He YL, Ding N, Xue HD, editors. Cyclic Changes of the Female Reproductive System in Young and Middle-aged Women during the Menstrual Cycle: an Initial 3T MRI Study based on T2 3DSpace Sequence. Proceedings of the 100th Annual Meeting of ESR: Vienna, 2014. (abstract 2939)

18. He Y, Ding N, Li Y, Li Z, Xiang Y, Jin Z, et al. H. 3-T diffusion tensor imaging (DTI) of normal uterus in young and middleaged females during the menstrual cycle: evaluation of the cyclic changes of fractional anisotropy (FA) and apparent diffusion coefficient (ADC) values. Br J Radiol 2015;88:20150043. Epub 2015 Mar 18. [CrossRef]
19. Fornasa F, Montemezzi S. Diffusion-weighted magnetic resonance imaging of the normal endometrium: temporal and spatial variations of the apparent diffusion coefficient. Acta Radiol 2010;53:586-90. [CrossRef]

20. Tsili AC, Argyropoulou MI, Tzarouchi L, Dalkalitsis N, Koliopoulos G, Paraskevaidis E, et al. Apparent diffusion coefficient values of the normal uterus: Interindividual variations during menstrual cycle. Eur J Radiol 2012;81:1951-6. [CrossRef]

21. Roberts DK, Lavia LA, Horbelt DV, Walker NJ. Changes in nuclear and nucleolar areas of endometrial glandular cells throughout the menstrual cycle. Int J Gynecol Pathol 1989;8:36-45. [CrossRef]

22. Christian M, Marangos P, Mak I, McVey J, Barker F, White J, et al. Interferon-gamma modulates prolactin and tissue factor expression in differentiating human endometrial stromal cells. Endocrinology 2001;142:3142-5. [CrossRef]

23. Song JY, Fraser IS. Effects of progestogens on human endometrium. Obstet Gynecol Surv 1995;50:385-94. [CrossRef] 